\title{
Stammzellen aus Plazentarestblut
}

\author{
R. Winter, Graz H. Hepp, München U. Haller, Zürich
}

\begin{abstract}
Zusammenfassung
Das Plazentarestblut enthält Stammzellen, die für die allogene Transplantation und in Zukunft möglicherweise auch für die autologe Anwendung Verwendung finden. Der Geburtshelfer wird damit in die Herstellung eines potentiellen Therapeutikums eingebunden. Die Arbeitsgruppe für Stammzelltransplantation der Österreichischen Gesellschaft für Hämatologie und Onkologie und die Österreichische Gesellschaft für Blutgruppenserologie und Transfusionsmedizin werden Richtlinien für die Gewinnung, Lagerung, Manipulation und Anwendung von Plazentarestblut erlassen, die für den Geburtshelfer von medizinischer und forensischer Bedeutung sind. Mit diesen geplanten Richtlinien der wissenschaftlichen Gesellschaften werden für den Fall der autologen Verwendung von Stammzellen Massnahmen getroffen, noch bevor eine medizinische Indikation für deren Einsatz besteht.
\end{abstract}

\section{Stem Cells from Cord Blood}

Cord blood contains hematopoietic stem cells that can be used for allogenic transplantation. It is expected that stem cells will also be used for autologous transplantation. Therefore, the obstetrician will be involved in the preparation of a potential therapeutic agent. The study group for stem cell transplantation of the Austrian Society of Hematology and Oncology as well as the Austrian Society for Blood Group Serology and Transfusion Medicine will publish guidelines for the production, storage, manipulation and application of cord blood. These guidelines will have medical and forensic significance for the obstetrician. With this project of the scientific societies, preventive measures for autologous transplantation will be instituted even before the existence of its medical indication.

Copyright $\odot 2002$ S. Karger AG, Basel
Hämatopoetische Stammzellen können aus dem Knochenmark oder peripheren Blut, aus Plazentarestblut oder aus embryonalem Gewebe gewonnen werden. Die Verwendung und Gewinnung embryonaler Stammzellen ist in Österreich wie in anderen Ländern derzeit gesetzlich verboten. Der Geburtshelfer wird mit dem Thema «Stammzellen» konfrontiert, da sie durch Punktion der vormals achtlos entsorgten Plazenta bzw. der Nabelschnur gewonnen werden können. Obwohl die Geburtshelfer derzeit keinen Bedarf für die Weiterverwendung von Stammzellen anmelden, sind sie in die Herstellung eines potentiellen Therapeutikums eingebunden.

Die Besitzverhältnisse sind bei autologer Verwendung klar und durch ein vertragliches Dauerrechtsverhältnis zu regeln, durch das die Mutter zugunsten des Kindes einen Betrag für etwaige künftige Leistungen bezahlt. Im Moment des Vertragsabschlusses ist allerdings noch nicht klar, ob, wann und unter welchen Umständen die Stammzellen des Plazentarestblutes dem Kind zugute kommen werden.

Bei allogener Verwendung der Stammzellquelle genügt die Zustimmung der Mutter im Namen des Kindes. Mit dieser Zustimmungserklärung übernehmen weder Mutter noch Kind irgendwelche Haftungen für den Fall, dass das Plazentarestblut genetische oder infektiöse Krankheitserreger enthält.

Wird hingegen das Plazentarestblut für die Forschung verwendet, so ist die Mutter über das Vorhaben zu informieren und ihr Einverständnis für die zweckgebundene Verwendung einzuholen.

Die Arbeitsgruppe für Stammzelltransplantation der Österreichischen Gesellschaft für Hämatologie und On-

Prof.Dr.R.Winter, Geburtshilflich-gynäkologische Universitätsklinik Auenbruggerplatz 14, A-8036 Graz (Österreich)

Tel. +43316385 2201, Fax +433163853061

E-Mail obstet.gynecol@kfunigraz.ac.at 
kologie und die Österreichische Gesellschaft für Blutgruppenserologie und Transfusionsmedizin werden Richtlinien für die Gewinnung, Lagerung, Manipulation und Anwendung von Plazentarestblut zur autologen Verwendung erlassen, die für den Geburtshelfer wichtige Passagen enthalten.

\section{Verantwortung und Zuständigkeit des}

Entnahmezentrums

Im Entnahmezentrum ist der Geburtshelfer verantwortlich für

- die Feststellung der Eignung der Schwangeren sowie des Kindes zur Spende

- die Aufklärung über die Spende

- die Einholung des schriftlichen Einverständnisses

- die korrekte Entnahme des Plazentarestblutes nach der schriftlichen Arbeitsanweisung der Plazentarestblutbank

\section{Aufklärung und Einverständniserklärung bei}

autologer Plazentarestblutspende

Die Einverständniserklärung soll folgende Punkte beinhalten

- die freiwillige und gegen Barzahlung vereinbarte Entnahme des Plazentarestblutes nach komplikationsloser Geburt und Abnabelung zum Zweck der Transplantation von hämatopoetischen Stammzellen; dabei sollte darauf hingewiesen werden, dass es sich bei der Blutentnahme um eine Restblutabnahme aus der Plazenta handelt und dieses Blut für das Kind nicht benötigt und normalerweise verworfen wird; weiters ist die Schwangere darüber zu informieren, dass nach derzeitigem medizinischem Wissensstand eine Transplantation von Plazentarestblutzellen ausserhalb allogener Indikationen nicht vertretbar ist, es insbesondere derzeit keine Indikation für eine autologe Stammzelltransplantation gibt und etwaige anderweitige Weiterverwendungen rein spekulativ sind und sich ausschliesslich auf erste tierexperimentelle Forschungsergebnisse stützen

- Entnahme von $20 \mathrm{ml}$ Blut der Mutter innerhalb von $48 \mathrm{~h}$ vor oder nach der Geburt zur Bestimmung der Gewebemerkmale sowie der notwendigen Infektionsmarker einschliesslich Hepatitis B und C mittels PCR-Einzeltestung und HIV-Testung

- anonymisierte Dokumentation der Daten und der beschriebenen Laboruntersuchungen des PlazentarestblutPräparates unter Beachtung des Datenschutzes

- bei der autologen Plazentarestblutspende wird das Nabelschnurpräparat ordnungsgemäss nach Rücksprache mit der das Kind vertretenden Mutter entsorgt, sobald der im Vertrag festgelegte Zeitraum verstrichen ist; auf Wunsch der das Kind vertretenden Mutter wird das Plazentarestblut-Präparat jederzeit vor Ablaufen der vereinbarten Frist verworfen

\section{Voraussetzungen für eine autologe}

Plazentarestblutentnahme

Die Untersuchung der Spendertauglichkeit umfasst eine ausführliche Anamnese und eine körperliche Untersuchung der Gebärenden. Es muss frühzeitig festgestellt und dokumentiert werden, ob sich die jeweilige Entbundene für eine Plazentarestblut-Spende eignet. Die Nabelschnur darf nicht früher oder später als gewöhnlich abgeklemmt werden. Eine Abnahme bei einer Mehrlings- oder Zwillingsgeburt ist nur dann zulässig, wenn das Abnahmeteam sicherstellt, dass Gewinnung, Zwischenlagerung und Transport des jeweiligen Plazentarestblutes getrennt und verwechslungsfrei erfolgen und das Plazentarestblut der Kinder nicht vermischt wird.

Ausschlusskriterien sind:

- Infektionskrankheiten der Schwangeren, die zu einer perinatalen Infektion des Neugeborenen führen können

- schwere Komplikationen der Spätschwangerschaft

- schwere perinatale Asphyxie des Neugeborenen

- Frühgeburtlichkeit bzw. pränatale Dystrophie mit einem Geburtsgewicht unter $1500 \mathrm{~g}$

\section{Plazentarestblut-Stammzellgewinnung}

Die Entnahme des Plazentarestblutes erfolgt gemäss der schriftlichen Arbeitsanweisung der Plazentarestblutbank im Kreisssaal des Entnahmezentrums oder nach Entwicklung der Plazenta in einem eigens hierzu bestimmten Raum unter Beachtung der sterilen Kautelen.

Für die Abnahme von Plazentarestblut ist durch die Plazentarestblutbank ein Schulungsprogramm zu etablieren. Als Mindesterfordernisse für Mitarbeiter und Mitarbeiterinnen des Abnahmeteams sind die jährliche Absolvierung einer facheinschlägigen Schulung sowie die abgeschlossene Ausbildung zur Hebamme, Diplom-Gesundheits-Krankenschwester oder medizinisch-technischen Assistentin erforderlich. Die Letztverantwortung über die korrekte Entnahme des Plazentarestblutes nach der schriftlichen Arbeitsanweisung der Plazentarestblutbank obliegt jedoch dem die Geburt leitenden Geburtshelfer.

5. Dokumentation bei autologer Plazentarestblut-

Spende

Folgende Daten sollen bei der Plazentarestblut-Gewinnung dokumentiert werden:

- Einverständniserklärung

- Entbindende: Alter, Blutgruppe (A, B, 0/Rhesus), Schwangerschaftskomplikationen, klinischer Status zum Zeitpunkt der Entbindung

- Neugeborenes: Zeitpunkt der Geburt, errechneter Termin bzw. Schwangerschaftswoche, Art der Entbindung, Komplikationen bei der Entbindung, Geschlecht, Geburtsgewicht, Geburtslänge, Apgar

- Protokoll über die Entnahme des Restblutes 


\section{Behältnisse und Beschriftung}

Zur Entnahme von Nabelschnurblut werden international zertifizierte, den jeweilig geltenden Richtlinien entsprechende sterile pyrogenfreie und geschlossene Behältnisse mit geeignetem Antikoagulans verwendet. Die Beschriftung muss die eindeutige Zuordnung des Plazentarestblutes gewährleisten.

\section{Lagerung und Transport}

Das gewonnene Plazentarestblut muss sachgerecht bei Raumtemperatur $\left(20-24^{\circ} \mathrm{C}\right)$ gelagert und transportiert werden.
Der Transport zum Verarbeitungszentrum soll innerhalb von $24 \mathrm{~h}$ erfolgen.

Wenn die zitierten Richtlinien in Kraft treten, werden Plazentarestblut-Banken und Geburtshelfer mehr als bisher in die Verantwortung genommen. Im Hinblick auf die autologe Verwendung von Stammzellen aus dem Plazentarestblut ist der seltene Fall eingetreten, dass wissenschaftliche Gesellschaften Richtlinien erarbeiten, noch bevor eine medizinische Indikation für deren Einsatz besteht.

Diese Richtlinien können als Vertrauen der Fachgesellschaften in die künftigen Ergebnisse der Stammzellforschung gewertet werden.

\section{Jahrestagung der Österreichischen Gesellschaft für Gynäkologie und Geburtshilfe (ÖGGG)}

29. Mai-1. Juni 2002, Alpbach, Tirol, Österreich

Auskunft: Frau Stefanie Leutgeb

KH Barmherzige Schwestern

Seilerstätte 4, A-4010 Linz (Österreich)

Tel. +437327677 7160, Fax +437327677 7676

\section{Jahresversammlung der Schweizerischen Gesellschaft für Gynäkologie und Geburtshilfe (SGGG)}

27.-29. Juni 2002, Interlaken, Schweiz

Auskunft: BV Consulting, Frau Barbara Vetsch Mooscherstrasse 21, CH-8804 Au (Schweiz)

Tel. +4116831484, Fax +4116831483

E-Mail kongresse@bvconsulting.ch, Web: http://www.bvconsulting.ch/kongresse 